\title{
EDITORIAL
}

\section{SCREENING FOR SARCOPENIA}

\author{
J.E. MORLEY, A.M. SANFORD

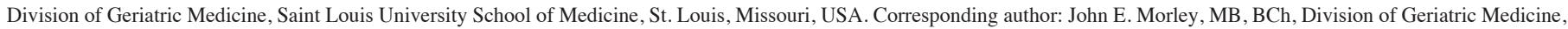 \\ Saint Louis University School of Medicine, 1402 S. Grand Blvd., M238, St. Louis, MO 63104, Email: john.morley@health.slu.edu
}

Key words: Sarcopenia, frailty, muscle mass.

“Tell your readers to use it or lose it. If you don't use your muscles, they get weak. If you don't use your mind it begins to fail." John Templeton

Sarcopenia, derived from the Greek term meaning "poverty of flesh," was first described by Irwin Rosenberg in the 1980s (1). Originally conceived as a loss of muscle mass in an older adult, the definition evolved and in 2010, it was redefined as the loss of muscle function or strength in the presence of low lean body mass $(2,3)$. In 2018 , it was officially recognized as a disease by the International Classification of Diseases - 10 (ICD-10) and an ICD-10 code was created for billing purposes (4). At the present time, the definition requires the presence of decreased grip strength or walking speed coupled with a measurement of lean mass, which is usually calculated by dual energy x-ray absorptiometry (DEXA). However, it has been recognized that normative values differ depending on the ethnicity of the individual (5). Recent studies have suggested that DEXA is an inaccurate measure of muscle strength, and that the D3-Creatine (D3-Cr) dilution is a more accurate measure of skeletal mass and may be more associated with health outcomes (i.e. functional status, falls, etc.) $(6,7)$.

There are two other forms of sarcopenia that are also widely recognized. The first is secondary sarcopenia, which occurs in persons with chronic diseases, e.g., diabetes mellitus, but does not meet the criteria for cachexia (8) The second is sarcopenic obesity, where the excess adipose tissue masks the loss of muscle, but yet the loss of muscle results in profound loss of strength and function (9). There are multiple causes of sarcopenia including inactivity, atherosclerosis, nerve dysfunction leading to muscle loss, cytokine excess occurring in inflammatory states, and loss of testosterone occurring in male hypogonadism (10). With aging, sarcopenia is a major cause of functional deterioration, hospitalization, falls, hip fracture, nursing home admission and overall mortality $(11,12)$.

Despite the clear deleterious effects of sarcopenia, the majority of clinicians appear unaware of its existence and rarely make the diagnosis (13). For busy clinicians to make a diagnosis they need simple screening tools that can be quickly

Table 1

SARC-F Screen for Sarcopenia

\begin{tabular}{lll}
\hline Component & Question & Scoring \\
\hline Strength & How much difficulty do you have in lifting and carrying 10 pounds? & $\begin{array}{l}\text { None }=0 \\
\text { Some }=1 \\
\text { A lot or unable }=2\end{array}$ \\
& & None $=0$ \\
Assistance in walking & How much difficulty do you have walking across a room? & Some $=1$ \\
& & A lot, use aids, or unable $=2$
\end{tabular}

Adapted from: Malmstrom TK, Morley JE. SARC-F: A simple questionnaire to rapidly diagnose sarcopenia. J Am Med Dir Assoc 2013;14:531. 


\section{THE JOURNAL OF NUTRITION, HEALTH \& AGING@}

performed. In 2013, we developed the 5-item SARC-F as a simple questionnaire to detect persons at risk for sarcopenia (Table 1) (14). Subsequently, it has been shown to have high specificity to predict functional deterioration, hospitalization, quality of life, and mortality (15-33). It also appears to be a reasonable indicator of sarcopenia based on the consensus definitions of sarcopenia (34). The sensitivity of the SARC-F is not as good as its specificity, but can be improved by combining the questionnaire with a measurement of calf circumference $(35,36)$. Woo et al $(37,38)$ found that using only 3 items (strength, ability to climb stairs and need for assistance in walking) of the 5 item questionnaire improved the diagnostic area under the curve values and had the highest predictive value of adverse outcomes. Based on the available data, SARC-F appears to be an excellent, brief screening tool for sarcopenia that can be easily utilized in busy clinical practices.

Another rapid screen for sarcopenia is the Short Portable Sarcopenia Measure (SPSM) (39), developed in 2009. It takes approximately 12 minutes to complete and consists of 3 components: lean muscle mass measurements calculated from the Tanita scale's bioelectrical impendence program, grip strength adjusted for height, and 5 chair stands. The SPSM showed convergent, discriminant, and predictive validity when compared to DEXA measures of muscle mass and alternative measures of muscle function. The SPSM has been found to have more sensitivity than the SARC-F, but does take longer and requires equipment to administer (40).

Rossi et al (41) developed the 5-item Mini-Sarcopenia Risk Assessment (MRSA-5) in 2017, which asks questions regarding age, activity level, meal consumption, hospitalizations, and weight loss in the past year. It was found to have good sensitivity, but less specificity for diagnosing sarcopenia. The MRSA-5 was compared with the SARC-F for identifying sarcopenia in a population of Chinese community-dwelling elders and was found to have a similar diagnostic accuracy with better sensitivity, but less specificity than the SARC-F (42). This screening tool at the present time needs further validation of its diagnostic validation.

In 2014, Ishii et al (43) developed a sarcopenia screen using age, grip strength and calf circumference. This measurement, validated in Japanese community-dwelling elders, shows high accuracy (both sensitivity and specificity and positive and negative predictive values) for sarcopenia. Its usefulness may be limited because of the time-consuming complex calculations required to ascertain a score.

In conclusion, the SARC-F is the easiest available measure for screening in the clinic and requires no special equipment. It has been recommended for screening for sarcopenia by the European Working Group on Sarcopenia in Older People (44), the International Conference on Sarcopenia and Frailty Research (ICSFR) (34) and the Society of Sarcopenia, cachexia and Wasting Disorders (45). With the availability of a rapid screening test for sarcopenia, it is suggested that screening occur on a yearly basis in all adults 65 years and older. Those who screen positive should receive a course of physical therapy and then strongly be encouraged to remain in an exercise group for the rest of their life (46-48). Recent data supports the use of exercise therapy even when in the hospital (49). Furthermore, there is some data supporting the use of a leucine enriched high protein supplement for added results, and while the benefits are controversial, there is likely no harm (50-55). Additionally, persons who do not go in the sunlight and have a low bioavailable vitamin $\mathrm{D}$ should receive adequate vitamin $\mathrm{D}$ supplementation $(56,57)$.

Disclosures: The authors declare there are no conflicts.

\section{References}

1. Rosenberg IH. Sarcopenia: Origins and clinical relevance. J Nutr 1997;127:99059915.

2. Cruz-Jentoft AJ, Baeyens JP, Bauer JM, et al. Sarcopenia: European consensus on definition and diagnosis: Report of the European Working Group on sarcopenia in older people. Age Ageing 2010;39:412-423.

3. Fielding RA, Vellas B, Evans WJ, et al. Sarcopenia: An undiagnosed condition in older adults. Current consensus definition: Prevalence, etiology, and consequences. International Working Group on Sarcopenia. J Am Med Dir Assoc 2011;12:249-256.

4. Anker SD, Morley JE, von Haehling S. Welcome to the ICD-10 code for sarcopenia. J Cachexia Sarcopenia Muscle 2016;7:512-514.

5. Chen L-K, Liu L-K, Wo J, et al. Sarcopenia in Asia: Consensus report of the Asian Working Group for Sarcopenia. J Am Med Dir Assoc 2014;15:95-101.

6. Evans WJ, Hellerstein M, Orwoll E, et al. D3-Creatine dilution and the importance of accuracy in the assessment of skeletal muscle mass. J Cachexia Sarcopenia Muscle 2019;10:14-21

7. Cawthon PM, Orwoll ES, Peters KE, et al; Osteoporotic Fractures in Men (MrOS) Study Research Group. Strong relation between muscle mass determined by D3-creatine dilution. Physical performance, and incidence of falls and mobility limitations in a prospective cohort of older men. J Gerontol A Biol Sci Med Sci 2019;74:844-852.

8. McKee A, Morley JE, Matsumoto AM, Vinik A. Sarcopenia: An endocrine disorder? Endocr Pract 2017;23:1140-1149.

9. Baumgartner RN, Wayne SJ, Waters DL, et al. Sarcopenia obesity predicts instrumental activities of daily living disability in the elderly. Obes Res 2004;12:1995-2004.

10. Morley JE. Pharmacologic options for the treatment of sarcopenia. Calcif Tissue Int 2016;98:319-333.

11. Morley JE. Frailty and sarcopenia: The new geriatric giants. Rev Invest Clin 2016;68:59-67.

12. Morley JE, Bauer JM. Editorial: The future of sarcopenia. Curr Opin Clin Nutr Metab Care 2019;22:1-3.

13. Cruz-Jentoft AJ, Sayer AA. Sarcopenia. Lancet 2019;393-:2636-2646.

14. Malmstrom TK, Morley JE. SARC-F: A simple questionnaire to rapidly diagnose sarcopenia. J Am Med Dir Assoc 2013;14:531-532.

15. Cao L, Chen S, Zou C, et al. A pilot study of the SARC-F scale on screening sarcopenia and physical disability in the. Chinese older people. J Nutr Health Aging 2014;18:277-283.

16. Woo J, Leung J, Morley JE. Validating the SARC-F: A suitable community screening tool for sarcopenia? J Am Med Dir Assoc 2014;15:630-634.

17. Malmstrom TK, Miller DK, Simonsick EM, et al. SARC-F: A symptom score to predict persons with sarcopenia at risk for poor functional outcomes. J Cachexia Sarcopenia Muscle 2016;7:28-36.

18. Liccini A, Malmstrom TK. Frailty and sarcopenia as predictors of adverse health outcomes in persons with diabetes mellitus. J Am Med Dir Assoc 2016;17:846-851.

19. Parra-Rodriguez L, Szlejf C, Garcia-Gonzalez AI, et al. Cross-cultural adaptation and validation of the Spanish-language version of the SARC-F to assess sarcopenia in Mexican Community-dwelling older adults. J Am Med Dir Assoc 2016;17:11421146

20. Ida S, Murata K, Nakadachi D, et al. Development of a Japanese version of the SARC-F for diabetic patients: An examination of reliability and validity. Aging Clin Exp Res 2017;29:935-942.

21. Tanaka S, Kamiya K, Hamazaki N, et al. Utility of SARC-F for assessing physical function in elderly patients with cardiovascular disease. J Am Med Dir Assoc 2017;18:176-181.

22. Rolland Y, Dupuy C, Abellan Van Kan G, et al. Sarcopenia screened by the SARC-F questionnaire and physical performances of elderly women: A cross-sectional study. J Am Med Dir Assoc 2017;18:848-852.

23. Kemmler W, Sieber C, Freiberger E, von Stengel S. The SARC-F questionnaire: 


\section{SCREENING FOR SARCOPENIA}

Diagnostic overlap with established sarcopenia definitions in older German men with sarcopenia. Gerontology 2017;63:411-416.

24. Ida S, Nakai M, Ito S, et al. Association between sarcopenia and mild cognitive impairment using the Japanese version of the SARC-F in elderly patients with diabetes. J Am Med Dir Assoc 2017;18:809.e9-809.e13.

25. Kim S, Kim M, Won CW. Validation of the Korean version of the SARC-F questionnaire to assess sarcopenia: Korean frailty and aging cohort study. J Am Med Dir Assoc 2018;19:40-45.e1.

26. Lim WS, Tay L, Yeo A, et al. Modulating effect of contextual factors on factor structure and reliability of SARC-F. J AM Med Dir Assoc 2018;19:551-553.

27. Ida S, Kaneko R, Murata K. SARC-F for screening of sarcopenia among older adults: A meta-analysis of screening test accuracy. J Am med Dir Assoc 2018;19:685-689.

28. Yang M, Hu X, Xie L, et al. Comparing mini sarcopenia risk assessment with SARC-F for screening sarcopenia in community-dwelling older adults. J Am Med Dir Assoc 2019;20:53-57.

29. Ida S, Murata K, Nakai M, et al. Relationship between sarcopenia and depression in older patients with diabetes: An investigation using the Japanese version of SARC-F. Geriatr Gerontol Int 2018;18:1318-1322.

30. Ida S, Kojima Y, Hamaoka S, et al. Validity of Japanese version of SARC-F questionnaire in patients with chronic liver disease. J Gastroenterol Hepatol 2019;34:947-953.

31. Bahat G, Yilmaz O, Kiliç C, et al. Performance of SARC-F in regard to sarcopenia definitions, muscle mass and functional measures. J Nutr Health Aging 2018;22:898903.

32. Su Y, Woo JW, Kwok TCY. The added value of SARC-F to prescreening using FRAX for hip fracture prevention in older community adults. J Am Med Dir Assoc 2019;20:83-89.

33. Yang M, Lu J, Jiang J, et al. Comparison of four sarcopenia screening tools in nursing home residents. Aging Clin Exp Res 2018;Dec 11. DOI: 10.1007/s40520-018-1083-x [Epub ahead of print]

34. Dent E, Morley JE, Crruz-Jentoft AJ, et al. International Cliical Practice Guidelines for Sarcopenia (ICFSR): Screening, diagnosis and management. J Nutr Health Aging 2018;22:1148-1161.

35. Barbosa-Silva TG, Menezes AM, Bielemann RM, et al; Grupo de Estudoes em Composição Corporal e Nutrição (COCONUT). Enhancing SARC-F; Improving sarcopenia screening in the clinical practice. J Am Med Dir Assoc 2016;17:11361141.

36. Bahat G, Oren MM, Yilmaz O, et al. Comparing SARC-F with SARC-CalF to screen sarcopenia in community living older adults. J Nutr Health Aging 2018;22:10341038.

37. Woo J, Yu R, Leung J. A 3-item SARC-F. J Am Med Dir Assoc 2018;19:223-228.

38. Yang M, Hu X, Xie L, et al. SARC-F for sarcopenia screening in communitydwelling older adults: Are 3 items enough? Medicine (Baltimore) 2018;97:e11726.

39. Miller J, Wells L, Nwulu U, et al. Validated screening tools for the assessment of cachexia, sarcopenia, and malnutrition: A systematic review. Am J Clin Nutr 2018;108:1196-1208.

40. Miller DK, Malmstrom TK, Andresen EM, et al. Development and validation of a short portable sarcopenia measure in the African American health project. J Gerontology: Med Sci 2009;64A:388-394.

41. Rossi AP, Micciolo R, Rubele S, et al. Assessing the risk of sarcopenia in the elderly:
The mini sarcopenia risk assessment (MSRA) questionnaire. J Nutr Helath Aging 2017;21:743-749.

42. Yang $\mathrm{M}, \mathrm{Hu} \mathrm{X}$, Xie $\mathrm{L}$, et al. Comparing mini sarcopenia risk assessment with SARC-F for screening sarcopenia in community-dwelling older adults. J Am Med Dir Assoc 2019;20:53-57.

43. Ishii S, Tomoki Tanaka, Shibasaki K, et al. Development of a simple screening test for sarcopenia in older adults. Geriatr Gerontol Int 2014;14 (Suppl 1): 93-101.

44. Cruz-Jentoft AJ, Bahat G, Bauer J, et al; Writing Group for the European Working Group on Sarcopenia in Older People 2 (EWGSP2), and the Extended Group for EWGSOP2. Sarcopenia: Revised European consensus on definition and diagnosis. Age Ageing 2019;48:16-31.

45. Bauer J, Morley JE, Schols AMWJ et al. Sarcopenia: A time for action. J Cachexia, Sarcopenia and Muscle. 2019 (In press).

46. Morley JE. Treatment of sarcopenia: The road to the future. J Cachexia Sarcopenia Muscle 2018;9:1196-1199.

47. Beckwée D, Delaere A, Aelbrecht S, et al. Exercise interventions for the prevention and treatment of sarcopenia: A systematic umbrella review. J Nutr Health Aging 2019;23:494-502.

48. Harada H, Kai H, Niiyama $\mathrm{H}$, et al. Effectiveness of cardiac rehabilitation for prevention and treatment of sarcopenia in patients with cardiovascular disease - a retrospective cross-sectional analysis. J Nutr Health Aging 2017;21:449-456.

49. Sáez de Asteasu ML, Martinez-Velilla N. Zamborn-Ferraresi F, et al. Physical exercise improves function in acutely hospitalized older patients: Secondary analysis of a randomized clinical trial. J Am Med Dir Assoc 2019;20:866-873.

50. Bauer J, Biolo G, Cederholm T, et al. Evidence-based recommendations for optimal dietary protein intake in older people: A position paper from the PROT-AGE study group. J Am Med Dir Assoc 2013;14:542-559.

51. Tieland M, Franssen R, Dullemeijer C, et al. The impact of dietary protein or amino acid supplementation on muscle mass and strength in elderly people: Individual participant data and meta-analysis of RCT's. J Nutr Health Aging 2017;21:994-1001.

52. Fielding RA, Travison TG, Kim DR, et al. Effect of structured physical activity and nutritional supplementation on physical function in mobility-limited older adults: Results from the VIVE2 randomized trial. J Nutr Health Aging 2017;21:936-942.

53. Osuka Y, Fujita S, Kitano N, et al. Effects of aerobic and resistance training combined with fortified milk on muscle mass, muscle strength, and physica performance in older adults: A randomized controlled trial. J Nutr Health Aging 2017;21:1349-1357.

54. Oktaviana J, Zanker J, Vogrin S, Duque G. The effect of $\beta$-hydroxy- $\beta$-methylbutyrate (HMB) on Sarcopenia and functional frailty in older persons: A systematic review. J Nutr Health Aging 2019;23:145-150.

55. Komar B, Schwingshackl L, Hoffmann G. Effects of leucine-rich protein supplement on anthropometric parameter and muscle strength in the elderly: A systematic review and meta-analysis. J Nutr Health Aging 2015;19:437-446.

56. Merchant RA, van Dam RM, Tan LWL, et al. Vitamin D binding protein and vitamin D levels in multi-ethnic population. J Nutr Health Aging 2018;22:1060-1065.

57. Morley JE. Editorial: Vitamin D: Does the emperor have no clothes? J Nutr Health Aging 2019;23:316-317. 